\title{
Fatores de risco associados à
}

Paulo César de Almeida ${ }^{3}$

EDWARD ARAujo JúNOR ${ }^{4}$

Helena Alves de Carvalho Sampaio ${ }^{5}$

\section{retenção de peso seis meses após o parto}

\author{
Risk factors associated with weight retention in postpartum period
}

\author{
Palavras-chave \\ Período pós-parto \\ Obesidade \\ Gravidez \\ Fatores de risco \\ Keywords \\ Postpartum period \\ Obesity \\ Pregnancy \\ Risk factors
}

\section{Resumo}

OBJETIVO: Identificar fatores de risco para retenção de peso em mulheres após o parto. MÉTODOS: Foi um estudo prospectivo observacional que acompanhou durante seis meses mulheres adultas que realizaram o parto em uma maternidade terciária. Aplicou-se um questionário estruturado antes da alta hospitalar e no período de seis semanas e seis meses após o parto, por meio de visitas domiciliares. $\bigcirc$ desfecho considerado foi retenção de peso após o parto (se risco >7,5 kg). As variáveis analisadas foram: idade, cor da pele, trabalho na gravidez e atual, renda, escolaridade, estado civil, idade da menarca, idade da mãe no primeiro parto, paridade, tipo de parto, intervalo interpartal, peso pré-gravídico, ganho de peso gestacional, percentual de gordura corpórea e estado nutricional. A verificação dos dados se iniciou com análises bivariadas entre prevalência de retenção de peso aos 6 meses e as diversas covariáveis $(p<0,2)$. Foram calculados os valores da Odds Ratio (OR) bruta e seus respectivos intervalos de confiança de 95\% (IC95\%). A última etapa da análise compreendeu a regressão logística multivariada para controle dos fatores de confusão, com estimativa de OR e IC95\%. RESULTADOS: A frequência de retenção de peso $>7,5 \mathrm{~kg}$ após 6 meses após o parto foi de 15\%. Na análise bivariada, a retenção de peso se associou às seguintes variáveis: idade da menarca $<12$ anos $(O R=3,7 ; \mid$ IC95\% 1, 1-13,2), ganho de peso gestacional $\geq 16$ kg $\mid O R=5,8$; IC95\% $1,8-18,6)$, percentual de gordura corporal no início do seguimento >30\% (OR=5,0; IC95\% 1, 1-23,6) e estado nutricional 6 semanas após o parto $>25 \mathrm{~kg} / \mathrm{m}^{2} \mid \mathrm{OR}=7,7 ; \mathrm{IC} 95 \%$ 1,6-36, 1). A partir da análise multivariada, somente o ganho de peso gestacional excessivo (OR=74, 1; IC95\% 9,0-609,6) permaneceu como fator de risco. CONCLUSÃO: $\bigcirc$ ganho de peso excessivo durante a gestação deve ter atenção especial na assistência pré-natal, tendo em vista a sua associação com a retenção de peso e possível excesso ponderal em mulheres após o parto nessas mulheres.

\section{Abstract}

PURPOSE: To identify risk factors for weight retention in women after childbirth. METHODS: This was a prospective observational study that followed for six months adult women who delivered at a tertiary center. Were applied a structured questionnaire before hospital discharge and at six weeks and six months after childbirth, through home visits. The outcome was weight retention after childbirth (if risk >7.5 kg). The variables analyzed were: age, skin color, working during pregnancy, income, education, marital status, age at menarche, maternal age at first birth, parity, mode of delivery, birth interval, pre-pregnancy weight, gestational weight gain, percent body fat, and nutritional status. Data were first analyzed by bivariate analysis between prevalence of weight retention at six months and several covariates $(p<0.2)$. We then calculated the Odds Ratio (OR) and their respective gross confidence intervals of $95 \%(95 \% \mathrm{Cl})$ and finally performed multivariate logistic regression to control for confounding factors and to estimate the OR and 95\% Cl. RESULTS: The frequency of weight retention $>7.5 \mathrm{~kg}$ by 6 months after delivery was 15\%. In bivariate analysis, weight retention was associated with the following variables: age at menarche $<12$ years $(O R=3.7 ; 95 \% \mathrm{Cl} 1.1-13.2)$, gestational weight gain $\geq 16 \mathrm{~kg}(\mathrm{OR}=5.8 ; 95 \% \mathrm{Cl} 1.8-18.6)$, percent body fat at baseline $>30 \%(O R=5.0 ; 95 \% \mathrm{Cl} 1.1-23.6)$, and nutritional status by 6 weeks postpartum $>25 \mathrm{~kg} / \mathrm{m}^{2} \mid \mathrm{OR}=7.7$; $95 \% \mathrm{Cl} 1.6-36.1$ ). In multivariate analysis, only excessive gestational weight gain (OR=74. 1; 95\% Cl 9.0-609.6) remained as a risk factor. CONCLUSION: Excessive weight gain during pregnancy should receive special attention in prenatal care in view of its association with weight retention and excess weight in women after childbirth.
Correspondência

Edward Aravio Júnior Departamento de Obstetrícia, Escola Paulista de Medicina, Universidade Federal de São Paulo Rua Napoleão de Barros, 875 - Vila Clementino CEP: 04024-002

São Paulo (SP), Brasil

Recebido

$17 / 02 / 2014$

Aceito com modificacọoes $28 / 03 / 2014$
Universidade Estadual do Ceará - UECE - Fortaleza (CE), Brasil.

IPrograma de Pós-Graduação em Saúde Pública, Universidade Estadual do Ceará - UECE; Professora do Curso de Nutrição, Centro de Ciências da Saúde, Universidade de Fortaleza - UNIFOR - Fortaleza (CE), Brasil.

2Department of Perinatal Medicine, Royal Women's Hospital and Department of Obstetrics and Gynaecology, University of MelbourneMelbourne, Australia.

${ }_{3}^{3}$ Programa de Mestrado Acadêmico em Saúde Pública, Universidade Estadual do Ceará - UECE - Fortaleza (CE), Brasil. ${ }^{4}$ Departamento de Obstetrícia, Escola Paulista de Medicina, Universidade Federal de São Paulo - UNIFESP - São Paulo (SP), Brasil. 5Programa de Mestrado Acadêmico em Saúde Pública e Doutorado em Saúde Coletiva, Universidade Estadual do Ceará - UECE Fortaleza (CE), Brasil.

Conflito de interesses: não há 
Introdução

O período da gestação e os primeiros meses após o parto têm sido identificados como momentos de risco na vida da mulher, devido à exposição a fatores que podem levar ao desencadeamento da obesidade ${ }^{1}$. Entre esses fatores, a retenção de peso após o parto tem sido apontada. Tal retenção de peso corresponde à diferença entre o peso após o parto e o peso pré-gestacional ${ }^{2}$.

Em relação ao tempo após o parto no qual a retenção de peso tem sido avaliada, os estudos variam bastante, com períodos que vão desde 2 meses $^{3}$ até 21 anos após o parto ${ }^{4}$. A retenção de peso em níveis indesejáveis tem sido frequentemente apontada $\mathrm{a}^{5,6}$.

Vários fatores têm sido investigados pelo aumento do risco de retenção de peso no período após o parto ${ }^{2-6}$. Destes, o ganho de peso gestacional excessivo tem sido identificado como um dos principais determinantes ${ }^{3,4,7}$. Destaca-se que o ganho ponderal excessivo na gestação é um achado comum nas pesquisas ${ }^{4,7}$, o que parece refletir a qualidade da assistência pré-natal. Todas as mulheres deveriam ser informadas sobre o ganho de peso saudável na gestação.

Outros fatores que possivelmente predispõem a mulher à retenção de peso após o parto são: idade, lactação, estado nutricional pré-gravídico, situação marital, cor da pele, alimentação e atividade física. Entretanto, os estudos têm encontrado resultados contraditórios para a maioria dessas variáveis ${ }^{8}$.

Foram encontrados poucos estudos nacionais ${ }^{3,9} \mathrm{e}$, nesse sentido, existe uma lacuna importante na discussão dos determinantes da retenção de peso no Brasil. No entanto, a produção científica internacional abordando a temática já é bastante considerável ${ }^{7}$.

Diante dos dados expostos, este estudo pretendeu contribuir para identificar fatores de risco associados à retenção de peso após o parto em mulheres atendidas em uma cidade do Nordeste brasileiro.

\section{Métodos}

Trata-se de um estudo prospectivo observacional que envolveu uma coorte com seis meses de seguimento. A população do estudo envolveu mulheres que realizaram parto no Hospital Geral Dr. César Cals (HGCC), localizado na cidade de Fortaleza (CE), instituição de referência estadual na área de ginecologia e obstetrícia. O referido hospital é detentor do maior contingente de partos mensais realizados na cidade, conforme dados da Secretária de Saúde do Estado do Ceará, sendo sua clientela constituída por usuários do Sistema Único de Saúde (SUS). A presente pesquisa foi aprovada pelo Comitê de Ética em Pesquisa da Universidade Estadual do Ceará (UECE) e autorizações por escrito foram obtidas de todas as participantes do estudo.
A coleta de dados ocorreu no período de janeiro a agosto de 2006. Entre janeiro e fevereiro ocorreu a coleta no hospital em que foram captadas mulheres no pós-parto imediato (até 48 horas do parto), antes da alta hospitalar. No final de fevereiro as visitas domiciliares foram iniciadas.

O cálculo amostral revelou um total de 157 mulheres. Porém, considerando os critérios de inclusão adotados na coorte (idade $\geq 20$ anos, não apresentar doenças crônicas conhecidas, não ter tido gestação gemelar, ter idade gestacional ao parto $\geq 37$ semanas e morar no município de Fortaleza), os critérios de exclusão (não saber informar o peso pré-gestacional e não realizar as três etapas de seguimento) e o tempo entre a primeira entrevista no hospital e a necessidade de início do segundo seguimento (primeira visita domiciliar), não se atingiu a amostra pretendida.

Aceitaram participar do estudo 110 mulheres e todas ingressaram na coorte. A perda de seguimento foi de $8(9,1 \%)$ mulheres; além destas, 2 outras mulheres não foram encontradas na primeira e na segunda visitas domiciliares, respectivamente, o que totalizou uma amostra final de 100 mulheres.

Antes da alta hospitalar, as mulheres responderam a um questionário estruturado, além de informarem seu peso pré-gestacional e terem o peso atual e a altura aferidos. $\mathrm{Na}$ captação, ficavam agendadas as visitas domiciliares que foram realizadas 6 semanas e 6 meses após o parto, sempre ocorrendo confirmação da visita 48 horas antes.

Durante as visitas domiciliares, as mulheres responderam novamente a um questionário estruturado e tiveram peso, circunferência da cintura e do quadril aferidos. As pacientes foram questionadas sobre informações demográficas, socioeconômicas, reprodutivas, antropométricas e dietéticas.

Para aferição do peso e da altura foram utilizados: balança Tanita com medidor de gordura corporal BF 682 Family Model $^{\circledR}$, com capacidade de $150 \mathrm{~kg}$ e intervalo de $100 \mathrm{~g}$, e antropômetro da marca AlturExata ${ }^{\circledR}$, com capacidade de $2,13 \mathrm{~m}$ e intervalo de $0,5 \mathrm{~cm}$, respectivamente. Além disso, as mulheres foram avaliadas quanto à composição corporal pela leitura direta na balança digital citada. Todos os procedimentos foram realizados pela pesquisadora principal, Cláudia Machado Coelho Souza de Vasconcelos, utilizando técnicas padronizadas ${ }^{10}$.

Nas análises do estudo foi tomada como variável dependente a retenção de peso seis meses pós-parto, cujo valor foi dicotomizado em $\leq 7,5 \mathrm{~kg}$ e $>7,5 \mathrm{~kg}$, nível este de retenção considerado elevado, sendo, portanto, prejudicial à saúde?

Para avaliar a associação das covariáveis sobre a retenção de peso pós-parto foram feitas as seguintes categorizações: faixa etária ( $\geq 30,20$ - 29), cor da pele (negra, branca/parda), trabalho na gestação e no pós-parto (não, sim), renda em reais $(\leq 1.000,1.001-4.900)$, escolaridade ( $\leq$ ensino fundamental, > ensino fundamental), estado civil (vive com companheiro, solteira), idade da menarca $(<12, \geq 12$ anos), idade da mãe no primeiro parto ( $<23, \geq 23$ anos), paridade 
(até 3, $\geq 4$ filhos), tipo de parto (cesariana, normal), intervalo interpartal em meses $(<21, \geq 21)$, peso pré-gravídico $(\geq 56 \mathrm{~kg}$, $<56 \mathrm{~kg})$, estatura $(\geq 159 \mathrm{~cm},<159 \mathrm{~cm})$, gordura corporal no pós-parto ( $\geq 30 \%,<30 \%)$, Índice de Massa Corporal (IMC) pré-gravídico e no pós-parto $\left(\geq 25,<25 \mathrm{~kg} / \mathrm{m}^{2}\right)$. Os pontos de corte utilizados nas análises foram baseados nas categorias de risco, hipotetizadas e estudadas pelas pesquisas científicas realizadas até o momento.

A análise dos dados se iniciou com análises bivariadas entre prevalência de retenção de peso $>7,5 \mathrm{~kg}$ aos seis meses e as diversas covariáveis. Foram calculadas ORs brutas e seus respectivos intervalos de confiança de 95\% (IC95\%). A última etapa da análise compreendeu a regressão logística multivariada para controle dos fatores de confusão, com estimativas de OR e de IC95\%. As variáveis que haviam apresentado associação $(\mathrm{p}<0,2)$ com a variável dependente na análise bivariada foram incluídas nas análises multivariadas subsequentes. Para todas as análises o nível crítico de significância utilizado foi de $5 \%$.

Os dados foram coletados em formulário específico, transferidos para uma planilha do programa Excel 2007 (Microsoft Corp., Redmond, WA, USA) e analisados pelo programa estatístico SPSS versão 11.0 (SPSS Inc., Chicago, IL, USA).

\section{Resultados}

A coorte foi constituída por mulheres relativamente jovens, com média de idade de 27,9 anos. A maior parte
(85\%) era casada ou vivia em regime de união estável. Mais da metade das mulheres (51\%) informou renda familiar baixa (de até 2 salários mínimos) e aproximadamente um terço (34\%) apresentou baixa escolaridade, com apenas o ensino fundamental incompleto. A maioria apresentou baixa paridade, $78 \%$ tinham tido até 2 partos e $74 \%$ tiveram a primeira gravidez com 23 ou mais anos de idade. As mulheres da amostra final apresentaram um IMC pré-gravídico médio de $24,5 \mathrm{~kg} / \mathrm{m}^{2}$.

As perdas de seguimento apresentaram características semelhantes à amostra, com média de idade de 26,9 anos, a maioria (80\%) era casada ou vivia em união estável, a metade tinha renda familiar baixa, $80 \%$ tinham tido até 2 partos e $80 \%$ tiveram a primeira gestação após 23 anos. O IMC médio também foi semelhante $-24,3 \mathrm{~kg} / \mathrm{m}^{2}$. Entretanto, apresentaram maior probabilidade $(60 \%)$ de ter baixa escolaridade.

Uma retenção de peso $>7,5 \mathrm{~kg} 6$ meses após o parto ocorreu em $15 \%$ da amostra. A média de retenção de peso

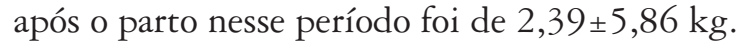

Dentre os fatores demográficos e socioeconômicos investigados pela possível associação com a retenção de peso $>7,5 \mathrm{~kg}$, foi constatada apenas tendência de risco aumentado para idade da mulher $\geq 30$ anos $(\mathrm{OR}=4,3 ; \mathrm{p}<0,05)$ na análise bivariada, não tendo sido constatadas outras associações. Para as demais variáveis deste bloco (renda, escolaridade e estado civil) não foram encontrados riscos maiores ou menores nas diferentes categorias investigadas (Tabela 1).

Tabela 1. Distribuição da amostra, segundo as variáveis demográficas e socioeconômicas e retenção de peso seis meses após o parto

\begin{tabular}{|c|c|c|c|c|c|c|}
\hline \multirow{2}{*}{ Variáveis } & \multicolumn{2}{|c|}{$>7,5 \mathrm{~kg}$} & \multicolumn{2}{|c|}{$\leq 7,5 \mathrm{~kg}$} & \multirow{2}{*}{ OR (IC95\%) } & \multirow{2}{*}{ Valor $\mathrm{p}$} \\
\hline & $n$ & $\%$ & $n$ & $\%$ & & \\
\hline \multicolumn{7}{|l|}{ Idade (anos) } \\
\hline$\geq 30$ & 13 & 20,3 & 51 & 79,7 & $4,3(0,9-20,4)$ & 0,06 \\
\hline $20-29$ & 2 & 5,6 & 34 & 94,4 & & \\
\hline \multicolumn{7}{|l|}{ Cor da pele } \\
\hline Negra & 1 & 16,7 & 5 & 83,3 & $1,1(0,1-10,5)$ & 0,9 \\
\hline \multicolumn{7}{|l|}{ Trabalho na gravidez } \\
\hline Não & 5 & 12,2 & 36 & 87,8 & $0,7(0,2-2,2)$ & 0,5 \\
\hline Sim & 10 & 16,9 & 49 & 83,1 & & \\
\hline \multicolumn{7}{|l|}{ Trabalho atual } \\
\hline Não & 3 & 10,3 & 26 & 89,7 & $0,6(0,1-2,2)$ & 0,4 \\
\hline \multicolumn{7}{|l|}{ Escolaridade } \\
\hline$\leq$ Fundamental & 7 & 15,6 & 38 & 84,4 & $1,1(0,4-3,2)$ & 0,8 \\
\hline$\geq$ Fundamental & 8 & 14,5 & 47 & 85,5 & & \\
\hline \multicolumn{7}{|l|}{ Estado civil } \\
\hline Vive com companheiro & 13 & 15,3 & 72 & 84,7 & $1,2(0,2-5,8)$ & 0,8 \\
\hline Solteira & 2 & 13,3 & 13 & 86,7 & & \\
\hline
\end{tabular}

OR: Odds Ratio; IC95\%: intervalo de confiança de 95\% 
Tabela 2. Distribuição da amostra, segundo associação de variáveis reprodutivas e do estado nutricional e retenção de peso seis meses após o parto

\begin{tabular}{|c|c|c|c|c|c|c|}
\hline \multirow{2}{*}{ Variáveis } & \multicolumn{2}{|c|}{$>7,5 \mathrm{~kg}$} & \multicolumn{2}{|c|}{$\leq 7,5 \mathrm{~kg}$} & \multirow{2}{*}{ OR (IC95\%) } & \multirow{2}{*}{ Valor $\mathrm{p}$} \\
\hline & n & $\%$ & $n$ & $\%$ & & \\
\hline \multicolumn{7}{|c|}{ Idade da menarca } \\
\hline$<12$ anos & 5 & 33,3 & 10 & 66,7 & $3,7(1,1-13,2)$ & 0,04 \\
\hline$\geq 12$ anos & 10 & 11,8 & 75 & 88,2 & & \\
\hline \multicolumn{7}{|c|}{ Idade da mãe $1^{\circ}$ parto } \\
\hline$<23$ anos & 9 & 16,4 & 46 & 83,6 & $1,3(0,4-3,89)$ & 0,6 \\
\hline \multicolumn{7}{|l|}{ Paridade } \\
\hline$\geq 4$ & 0 & 0,0 & 10 & 100,0 & - & - \\
\hline $1-3$ & 15 & 16,7 & 75 & 83,3 & & \\
\hline \multicolumn{7}{|l|}{ Tipo de parto } \\
\hline Cesária & 7 & 15,2 & 39 & 84,8 & $1,0(0,3-3,1)$ & 0,9 \\
\hline \multicolumn{7}{|c|}{ Peso pré-gravídico (kg) } \\
\hline$\geq 56$ & 7 & 12,7 & 48 & 87,3 & $0,7(0,2-2,0)$ & 0,4 \\
\hline$<56$ & 8 & 17,8 & 37 & 82,2 & & \\
\hline \multicolumn{7}{|c|}{ Ganho de peso (kg) } \\
\hline$\geq 16$ & 8 & 36,4 & 14 & 63,6 & $5,8(1,8-18,6)$ & 0,01 \\
\hline$<16$ & 7 & 9,0 & 71 & 91,0 & & \\
\hline \multicolumn{7}{|l|}{ Altura (cm) } \\
\hline$\geq 159$ & 4 & 14,8 & 23 & 85,2 & $1,0(0,3-3,4)$ & 0,9 \\
\hline$<159$ & 11 & 15,1 & 62 & 84,9 & & \\
\hline \multicolumn{7}{|c|}{$\%$ de gordura (imediato) } \\
\hline
\end{tabular}

OR: Odds Ratio; IC95\%: intervalo de confiança de 95\%; IMC: Índice de Massa Corporal

No tocante à cor da pele, não foi verificado no presente estudo risco aumentado de retenção de peso no pós-parto entre mulheres negras $(\mathrm{OR}=1,1 ; \mathrm{p}>0,05)$. Para as mulheres que retornaram ao trabalho no pós-parto não foi observado menor risco de reter peso no pós-parto $(\mathrm{OR}=0,6 ; \mathrm{p}>0,05)$.

A menarca precoce foi identificada como fator que aumenta o risco de reter peso no pós-parto $(\mathrm{OR}=3,7$; $\mathrm{p}<0,05)$, porém, após o controle para possíveis fatores de confusão, tal associação desapareceu. Para as outras variáveis reprodutivas não foram encontradas associações (Tabela 2).

$\mathrm{Na}$ análise bivariada, algumas variáveis estiveram associadas com a retenção de peso $>7,5 \mathrm{~kg}$ seis meses após o parto: ganho de peso na gestação atual $(\mathrm{OR}=5,8$; $\mathrm{p}<0,05)$, percentual de gordura corporal no pós-parto imediato $(\mathrm{OR}=5,0 ; \mathrm{p}<0,05)$ e o IMC de seis semanas após o parto $(\mathrm{OR}=7,7 ; \mathrm{p}<0,05)$.
Após análise multivariada, somente o ganho de peso na gestação atual permaneceu associado com a retenção em níveis elevados 6 meses após o parto (OR=74,1; $\mathrm{p}<0,05)$. Portanto, após o controle de possíveis fatores de confusão, somente mulheres com ganho de peso gestacional $\geq 16 \mathrm{~kg}$ tiveram risco maior de apresentar retenção ponderal elevada 6 meses pós-parto (Tabela 3).

\section{Discussão}

No presente estudo, a frequência de mulheres com nível de retenção de peso no período pós-parto considerado excessivo foi elevada, fato também observado em outro estudo, realizado em mulheres brasileiras acompanhadas durante nove meses pós-parto, que encontrou percentual parecido de mulheres com os mesmos níveis de retenção ${ }^{9}$. O estudo citado, apesar de envolver amostra 
Tabela 3. Regressão logistica para retenção de peso $>7,5 \mathrm{~kg} 6$ meses após o parto

\begin{tabular}{|c|c|c|c|}
\hline Variáveis & OR ajustada & IC95\% & Valor $p$ \\
\hline \multicolumn{4}{|c|}{ Idade (anos) } \\
\hline$\geq 30$ & 1,0 & $0,3-3,2$ & 0,9 \\
\hline \multicolumn{4}{|c|}{ 20-29 } \\
\hline \multicolumn{4}{|c|}{ Idade da menarca (anos) } \\
\hline$<12$ & 1,1 & $0,2-5,5$ & 0,8 \\
\hline \multicolumn{4}{|l|}{$\geq 12$} \\
\hline \multicolumn{4}{|c|}{ \% de gordura (pós-parto imediato) } \\
\hline$\geq 30$ & 0,4 & $0,1-2,9$ & 0,4 \\
\hline \multicolumn{4}{|l|}{$<30$} \\
\hline \multicolumn{4}{|c|}{ IMC pré-gravídico ( $\left.\mathrm{kg} / \mathrm{m}^{2}\right)$} \\
\hline$\geq 25$ & 1,6 & $0,3-9,9$ & 0,5 \\
\hline \multicolumn{4}{|l|}{$<25$} \\
\hline \multicolumn{4}{|c|}{ Ganho de peso (kg) } \\
\hline$\geq 16$ & 74,1 & $9,0-609,6$ & 0,01 \\
\hline$<16$ & & & \\
\hline
\end{tabular}

OR: Odds Ratio; IC95\%: intervalo de confiança de 95\%; IMC: Índice de Massa Corporal

maior e acompanhar as mulheres por um período mais longo, estabeleceu critérios de seleção da amostra parecidos: ambos utilizaram o peso pré-gravídico reportado e acompanharam clientes do SUS.

Em outros países são observados resultados semelhantes com amostras de tamanho parecido. Um estudo realizado em Amsterdam encontrou retenção de peso igual ou superior a $5 \mathrm{~kg}$ no período de um ano após o parto em $20 \%$ das 118 mulheres acompanhadas 5 . Para o mesmo período de tempo pós-parto, com amostra maior (413), observou-se na cidade de Nova Iorque frequência de retenção igual ou maior que $4,55 \mathrm{~kg} \mathrm{em} 24 \%$ da amostra ${ }^{2}$. $\mathrm{Na}$ Dinamarca, Rode et al. ${ }^{11}$, estudando uma amostra maior $(n=1.840)$, encontraram que $13,2 \%$ das mulheres retiveram $5 \mathrm{~kg}$ ou mais 1 ano após o parto.

Tais resultados revelam que no Brasil e em outras partes do mundo o acompanhamento do peso da mulher no período pós-parto possivelmente não tem recebido a devida atenção. Vários fatores têm sido investigados pela associação com a retenção de peso no período pós-parto. Dentre eles, destaca-se o ganho de peso gestacional excessivo.

No presente estudo, o ganho de peso excessivo permaneceu como fator associado à retenção de peso em níveis indesejáveis, seis meses após o parto. Esta variável é de longe um dos mais importantes fatores determinantes da retenção de peso no pós-parto apontados na literatura ${ }^{4,7,11,12}$. Um dos estudos prospectivos mais longos sobre o tema, realizado na Australia, avaliou 21 anos após o parto uma coorte de 2.055 mulheres e encontrou que o ganho de peso durante a gestação é um preditor independente de obesidade em mulheres. Um metanálise com 12 estudos realizada por Mannan et al. ${ }^{7}$ concluiu que o ganho de peso acima das recomendações do Institute of Medicine pode levar a mudanças de peso no pós-parto em curto e longo prazos.

Os estudos comparados utilizaram como referência para a análise do ganho de peso as recomendações do Institute of Medicine e para o cálculo do ganho de peso gestacional, o peso reportado pela mulher. Os resultados encontrados mostram a necessidade de estudos de intervenção randomizados controlados para controle do ganho de peso na gestação, como lembra Mamun et al. ${ }^{4}$.

As demais variáveis analisadas não foram identificadas como preditoras da retenção de peso após o parto, o que corrobora outros estudos, como é o caso da cor da pele ${ }^{12}$, renda familiar ${ }^{13,14}$, menarca precoce ${ }^{9}$, tipo de parto $^{3}$, estado nutricional pré-gravídico ${ }^{9,15}$, percentual de gordura corporal ${ }^{13}$. Por outro lado, outros estudos já apontaram idade ${ }^{9,16,17}$, cor da pele, desemprego de mulheres $^{18}$, renda familiar ${ }^{6,9,19}$, escolaridade ${ }^{5,9}$, estado civil $^{14,17}$, idade inferior a 23 anos no primeiro parto', paridade ${ }^{9}$ e estado nutricional pré-gravídico ${ }^{3,16,20}$ como fatores associados à retenção de peso após o parto.

Algumas limitações podem contribuir para a ausência de associação entre algumas variáveis. No caso da cor da pele, há pequena frequência de mulheres de cor negra nesse estudo. Portanto, diferenças culturais e étnicas podem influenciar os resultados controversos. Acredita-se que não somente questões genéticas possam interferir no risco aumentado para mulheres negras, mas que também questões sociais, comportamentais e culturais possam justificá-lo. Em relação à paridade, a pequena frequência de mulheres com paridade elevada pode ter contribuído para a ausência de associação no grupo, composto por mulheres relativamente jovens. É importante frisar que a mudança de peso relacionada à paridade é dependente do efeito da lactação, mas os resultados dos efeitos da lactação sobre a retenção de peso após a gestação têm sido inconclusivos, o que pode ser justificado pelas diferenças nos desenhos dos estudos, a definição da variável amamentação e a sua duração e intensidade, tempo de seguimento pós-parto e controle para fatores de confusão . $^{8}$

Desse modo, é possível inferir que as contradições observadas nos resultados dos diferentes estudos devem-se, provavelmente, às diferenças nos desenhos dos estudos, definição, mensuração e categorização de variáveis, tamanho da amostra, controle para fatores de confusão, tempo de seguimento pós-parto ${ }^{4,8}$.

Não se pode deixar de mencionar algumas limitações metodológicas deste estudo que devem ser consideradas para a interpretação dos dados. Dentre elas, a ausência de grupos de comparação incluindo mulheres não grávidas ou nulíparas, visando isolar o efeito da paridade no aumento do peso e aumentar a acurácia das estimativas. Entretanto, tal estratégia nem sempre é factível e não tem sido sistematicamente utilizada nos estudos. 
Além disso, o peso pré-gravídico reportado pela própria mulher também tem sido identificado como uma limitação importante, já que esse tipo de dado pode gerar viés nas estimativas de mudança de peso no pós-parto, superestimando os valores de retenção. O ideal seria medir o peso logo após a concepção, o que, em termos práticos, é pouco exequível. Por outro lado, outros estudos já encontraram uma alta correlação entre o peso pré-gestacional reportado e o peso efetivamente aferido. A maioria dos estudos usou o peso pré-gestacional reportado ${ }^{4,5,9}$. Alguns utilizaram o peso no primeiro trimestre de gestação ${ }^{17}$. E, finalmente, a amostra relativamente pequena, apesar de haver outros estudos que trabalharam com amostras semelhantes e até menores.

Apesar das limitações metodológicas citadas, destaca-se a baixa taxa de perda de seguimento desse estudo (9,09\%), bem inferior às encontradas em outros estudos. Os dados apresentados mostram que foi significativa a frequência da retenção de peso no período pós-parto para esse grupo específico de mulheres residentes na cidade de Fortaleza (CE). O ganho de peso gestacional excessivo foi confirmado como um dos maiores fatores determinantes do peso da mulher no pós-parto.

Foi observado que o efeito de outros fatores ainda permanece controverso. Estudos com amostras maiores e com ajuste para fatores de confusão podem revelar outros fatores de risco não encontrados neste estudo. No entanto, considerando a produção científica disponível no momento e os resultados encontrados no presente estudo, é iminente a necessidade de políticas mais eficazes de intervenção nutricional mais eficazes, com acompanhamento permanente do peso e orientações alimentares, tanto na gestação quanto no período pós-parto, como forma de diminuir a magnitude da retenção de peso no pós-parto e os possíveis riscos associados.

\section{Agradecimentos}

A Taciane Câmara e Débora Férrer, pelo apoio na coleta de dados, e à Fundação Cearense de Apoio ao Desenvolvimento Científico e Tecnológico (FUNCAP), pela bolsa de mestrado concedida a Cláudia Machado Coelho Souza de Vasconcelos.

\section{Referências}

1. Sarwer DB, Allison KC, Gibbons LM, Markowitz JT, Nelson DB. Pregnancy and obesity: a review and agenda for future research. J Womens Health (Larchmt). 2006; 15(6):720-33.

2. Lipsky LM, Strawderman MS, Olson CM. Maternal weight change between 1 and 2 years postpartum: the importance of 1 year weight retention. Obesity (Silver Spring). 2012;20(7): 1496-502.

3. Rebelo F, Castro MB, Dutra CL, Schlussel MM, Kac G. [Factors associated with post-partum weight retention in a cohort of women, 2005-2007]. Rev Bras Saude Mater Infant. 2010;10(2):219-27. Portuguese.

4. Mamun AA, Kinarivala M, O'Callaghan M, Williams GM, Najman JM, Callaway LK. Associations of excess weight gain during pregnancy with long-term maternal overweight and obesity: evidence from 21 y postpartum follow-up. Am J Clin Nutr. 2010;91(5):1336-41.

5. Althuizen E, van Poppel MN, de Vries JH, Seidell JC, van Mechelen W. Postpartum behaviour as predictor of weight change from before pregnancy to one year postpartum. BMC Public Health. $2011 ; 11: 165$.

6. Pedersen P, Baker JL, Henriksen TB, Lissner L, Heitmann BL, Sørensen $\mathrm{TI}$, et al. Influence of psychosocial factors on postpartum weight retention. Obesity (Silver Spring). 2011 ; 19(3):639-46.

7. Mannan M, Doi SA, Mamun AA. Association between weight gain during pregnancy and postpartum weight retention and obesity: a bias-adjusted meta-analysis. Nutr Rev. 2013;71(6):343-52.

8. Castro MB, Kac G, Sichieri R. [Nutritional and socio-demographic determinants of post-partum weight change: a literature review]. Rev Bras Saude Mater Infant. 2009;9(2):125-37. Portuguese.

9. Kac G, Benício MH, Velásquez-Meléndez G, Valente JG. Nine months postpartum weight retention predictors for Brazilian women. Public Health Nutr. 2004;7(5):621-8.

10. World Health Organization. Physical status: the use and interpretation of anthropometry. Report of a WHO Expert Committee. Geneva: $\mathrm{WHO} ; 1995$.
1 1. Rode L, Kjærgaard H, Ottesen B, Damm P, Hegaard HK. Association between gestational weight gain according to body mass index and postpartum weight in a large cohort of Danish women. Matern Child Health J. 2012;16(2):406-13.

12. Melzer K, Schutz Y. Pre-pregnancy and pregnancy predictors of obesity. Int J Obes (Lond). 2010;34(Suppl 2):S44-S52.

13. Kac G, D'Aquino Benício MH, Valente JG, Velásquez-Meléndez G. Postpartum weight retention among women in Rio de Janeiro: a follow-up study. Cad Saúde Pública. 2003; 19(Suppl 1):S149-S61.

14. Jordão IS, Kac G. Determinantes da retenção de peso pós-parto segundo a cor da pele em mulheres do Rio de Janeiro, Brasil. Rev Panam Salud Pública. 2005; 18(6):403-1 1.

15. Valeggia CR, Ellison PT. Impact of breastfeeding on anthropometric changes in peri-urban Toba women (Argentina). Am J Hum Biol. $2003 ; 15(5): 717-24$

16. Krause KM, Lovelady CA, Peterson BL, Chowdhury N, Østbye T. Effect of breast-feeding on weight retention at 3 and 6 months postpartum: data from the North Carolina WIC Programme. Public Health Nutr. 2010;13(12):2019-26.

17. Olson CM, Strawderman MS, Hinton PS, Pearson TA. Gestational weight gain and postpartum behaviors associated with weight change from early pregnancy to 1 y postpartum. Int J Obes Relat Metab Disord. 2003;27(1):117-27.

18. Siega-Riz AM, Herring AH, Carrier K, Evenson KR, Dole N, Deierlein A. Sociodemographic, perinatal, behavioral, and psychosocial predictors of weight retention at 3 and 12 months postpartum. Obesity (Silver Spring). 2010;18(10):1996-2003.

19. Shrewsbury V, Robb KA, Power C, Wardle J. Socioeconomic differences in weight retention, weight-related attitudes and practices in postpartum women. Matern Child Health J. 2009; 13(2):23 1-40.

20. Baker JL, Gamborg M, Heitmann BL, Lissner L, Sørensen TI, Rasmussen KM. Breastfeeding reduces postpartum weight retention. Am J Clin Nutr. 2008;88(6):1543-51. 